\title{
Reaction of Acyclic Enaminones with Methoxymethylene Meldrum's Acid. Synthetic and Structural Implications ${ }^{\#}$
}

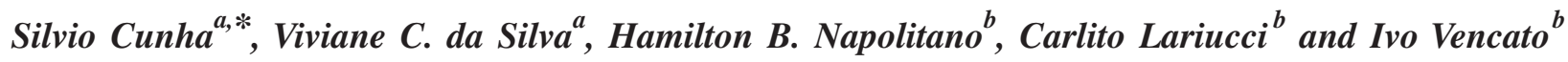 \\ ${ }^{a}$ Instituto de Química, ${ }^{b}$ Instituto de Física, Universidade Federal de Goiás, CP 131, 74001-970 Goiânia - GO, Brazil
}

\begin{abstract}
A reação de enaminonas com o derivado metoximetilênico do ácido de Meldrum forneceu $\mathrm{N}$-adutos e/ou $\mathrm{C}$-adutos das enaminonas, em rendimentos moderados a bons. A regioquímica da reação se revelou dependente do substituinte do nitrogênio da enaminona, e o $C$-aduto formado é precursor para 2-piridonas. A análise da difração de raios $\mathrm{X}$ de dois $N$-adutos revelou que estes adutos possuem a configuração $Z-s-Z$.
\end{abstract}

The reaction of acyclic enaminones with methoxymethylene Meldrum's acid afforded $N$-adduct and/or $C$-adduct of enaminones in moderate to good yields. The regiochemistry of this reaction depends on the $N$-amino substituent of the enaminone. The $C$-adduct is a precursor to 2-pyridones. $\mathrm{X}$-ray analysis of two $\mathrm{N}$-adducts were investigated and the $Z$-s- $Z$ configuration assigned.

Keywords: enaminones, Meldrum's acid, aza-annulation, 2-pyridone

\section{Introduction}

The fascinating chemistry of enaminones and their derivatives has attracted the attention of numerous researchers due to their ambiphilic and ambident properties and their potential in the synthesis of heterocyclic compounds. ${ }^{1}$ In this context, the aza-annulation reaction of cyclic and acyclic enaminones has been extensively used in the preparation of a broad spectrum of nitrogencontaining compounds, ${ }^{2}$ mainly in alkaloids ${ }^{3}$ and conformationally constrained peptide analogues. ${ }^{4}$ Because of these applications several protocols for the synthesis of enaminones have been developed. ${ }^{5}$ Among them, the solid support method developed by Braibante and co-workers ${ }^{6}$ and its systematic use in the synthesis of pyrazoles and isoxazoles derivatives is noteworthy. ${ }^{7}$

While the reaction of methoxymethylene Meldrum's acid (1) with cyclic enaminones has been documented (Scheme 1, reactions 1-4), ${ }^{8}$ much less study has been carried out with $\mathbf{1}$ and acyclic enaminones. There is only a single paper describing two examples of reaction of $\mathbf{1}$ with

* e-mail: silviodc@ufba.br

Present address: Instituto de Química, Universidade Federal da Bahia, Campus de Ondina, 40170-290 Salvador - BA, Brazil.

\# Dedicated to Professor Albert James Kascheres, a great mentor and pioneer in ciclopropenone chemistry in Brazil, on the occasion of his 60th birthday. enaminones 11 and 13a (Scheme 1, reactions 5-6). ${ }^{8}$ However, the aza-annulation of derivatives 12 and $\mathbf{1 4 a}$ under pyrolysis conditions (Scheme 1, reaction 7) is not synthetically efficient because mixture of products and poor yields are obtained. In search for a general method of synthesis of derivatives $\mathbf{1 2}$ to $\mathbf{1 4}$ we undertook a study concerning the reactions of acyclic enaminones and methoxymethylene Meldrum's acid (1). In this paper we report the results of this study with emphasis on synthetic, mechanistic and structural implications.

\section{Results and Discussion}

Enaminones may act as an ambident nucleophile by reaction at the nitrogen and at the $\beta$-carbon. The reactions of enaminones and methoxymethylene Meldrum's acid depend on the $N$-amino substituent, Scheme 1. C-Adducts are obtained with $\mathrm{NR}_{2}$ substituent $(\mathrm{R}=$ alkyl $)$ and $N$-adducts with the $\mathrm{NH}_{2}$ group. However, when we attempted the reaction of enaminone 13a with 1 under the literature condition $^{8}$ a low yield of the $N$-adduct 14a was obtained (36\%, instead of the reportedly obtained $60 \%$ yield). Additionally, a small quantity of the $C$-adduct 18a was isolated (3.2\% yield, Scheme 2 ), which was not previously reported. The spectral data of compound 14a here obtained were identical with those described. ${ }^{8}$ The ${ }^{1} \mathrm{H}$ NMR spectra contained a low field N-H (13.93 ppm) which suggests its participation in intramolecular hydrogen bonding. Despite 


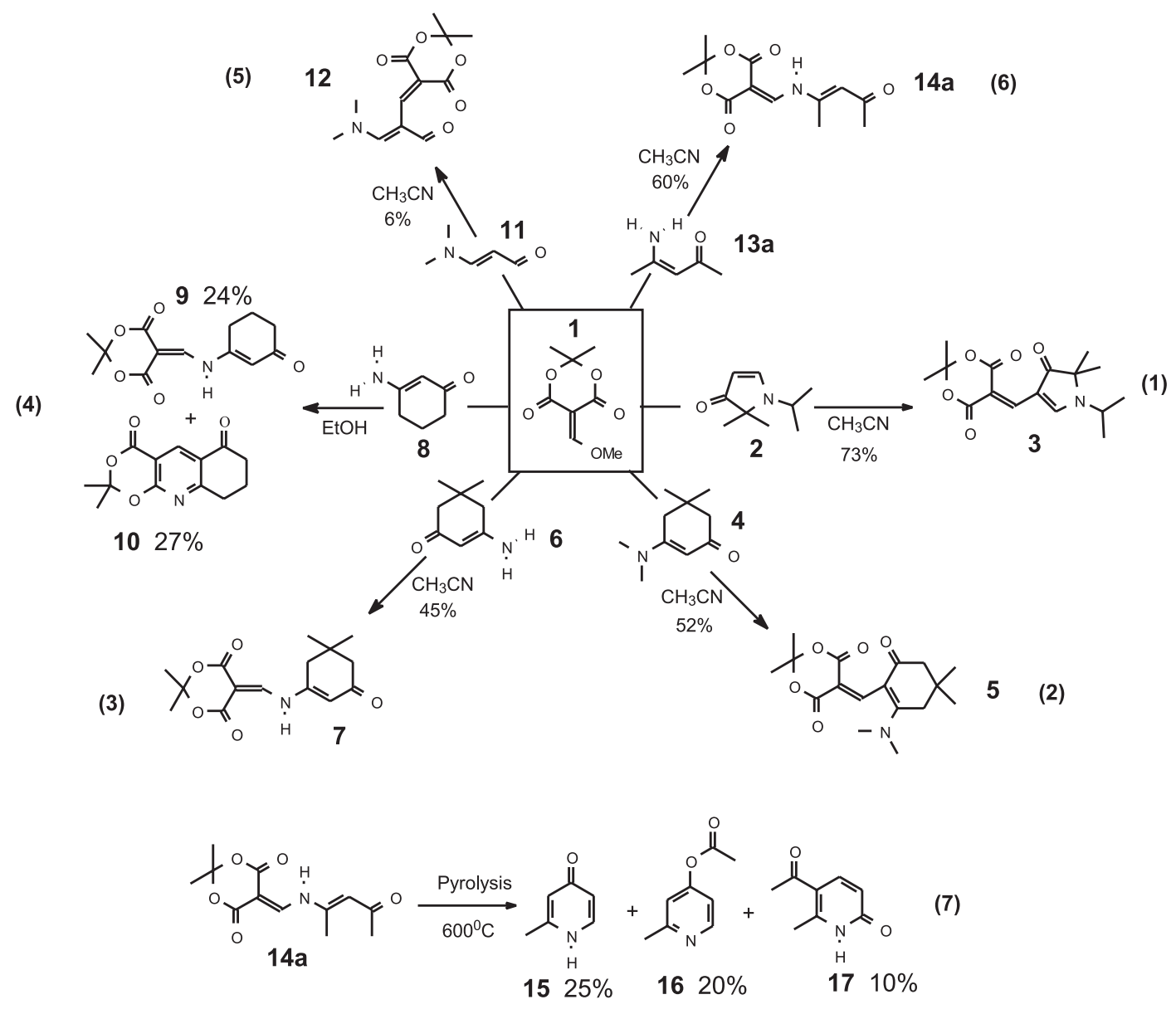

Scheme 1.

the reportedly $E-s-E$ configuration to $\mathbf{1 4 a}$ we assigned the $Z$-s- $Z$ configuration to the $N$-adduct because $E-s-E$ and $Z$ $s-Z$ configurational isomers of enaminones are well distinguished by typical N-H chemical shifts ( $E$-isomer: 4.1-6.5 ppm; Z-isomer: 9.5-12.0 ppm). ${ }^{9}$ Moreover, the structure of $\mathbf{1 4 a}$ was unambiguously confirmed by X-ray analysis and the $Z$ configuration corroborated, as shown in Figure 1.

In addition, extension of the reported protocol ${ }^{8}$ to other enaminones afforded complex mixtures. Better results were obtained when $\mathrm{CH}_{2} \mathrm{Cl}_{2}$ was used as solvent instead of $\mathrm{CH}_{3} \mathrm{CN}$ (Scheme 2). With this modification $\mathrm{N}$ - and $\mathrm{C}$ adducts 14a-b and 18a-b were obtained in a 2:1 ratio, respectively. With enaminone $\mathbf{1 3 c}$ only the $C$-adduct $\mathbf{1 8 c}$ was formed in good yield.

To our surprise, when we attempted the reaction of $\mathbf{1}$ with enaminone 13d a complex mixture was obtained, and the 2-pyridone 19d could be isolated in $28 \%$ yield (Scheme 3, reaction. 1). Unfortunately, the pyridone 19d was an unstable solid that precluded its complete spectral characterization. However, its structure could be assigned by comparison of its IR and ${ }^{1} \mathrm{H}$ NMR spectra with analogue 19c (see Experimental). The formation of 19d may be visualized as occurring through the aza-annulation of the initial $C$-adduct of the reaction of $\mathbf{1}$ and $\mathbf{1 3 d}$. To support this mechanistic proposal we decided to perform the thermolysis of the isolated $C$-adduct 18c. In this way, 18c was refluxed in toluene and the 2-pyridone 19c was obtained in good yield (Scheme 3, reaction 2). The structure of 19c was corroborated by analysis of a long-range heterocorrelation (COLOC) spectrum which showed correlation $\left({ }^{3} J\right)$ of the hydrogen at $\mathrm{C}-4$ with the carbonyl C- 2 and with C-6 as well as the other correlations indicated in Scheme 4, which also presents the mechanistic pathway to 19c. Interestingly, in this thermal cyclization the typical $\mathrm{CO}_{2}$ elimination from the methylene Meldrum's acid moiety was not observed. ${ }^{10}$ It should also be pointed out that the relative low temperature required to form the 2pyridones 19c-d makes this methodology synthetically attractive, contrasting with the literature pyrolysis condition for the $N$-adduct $\mathbf{1 4 a} .^{8}$

Understanding how enaminones fit together in the solid 

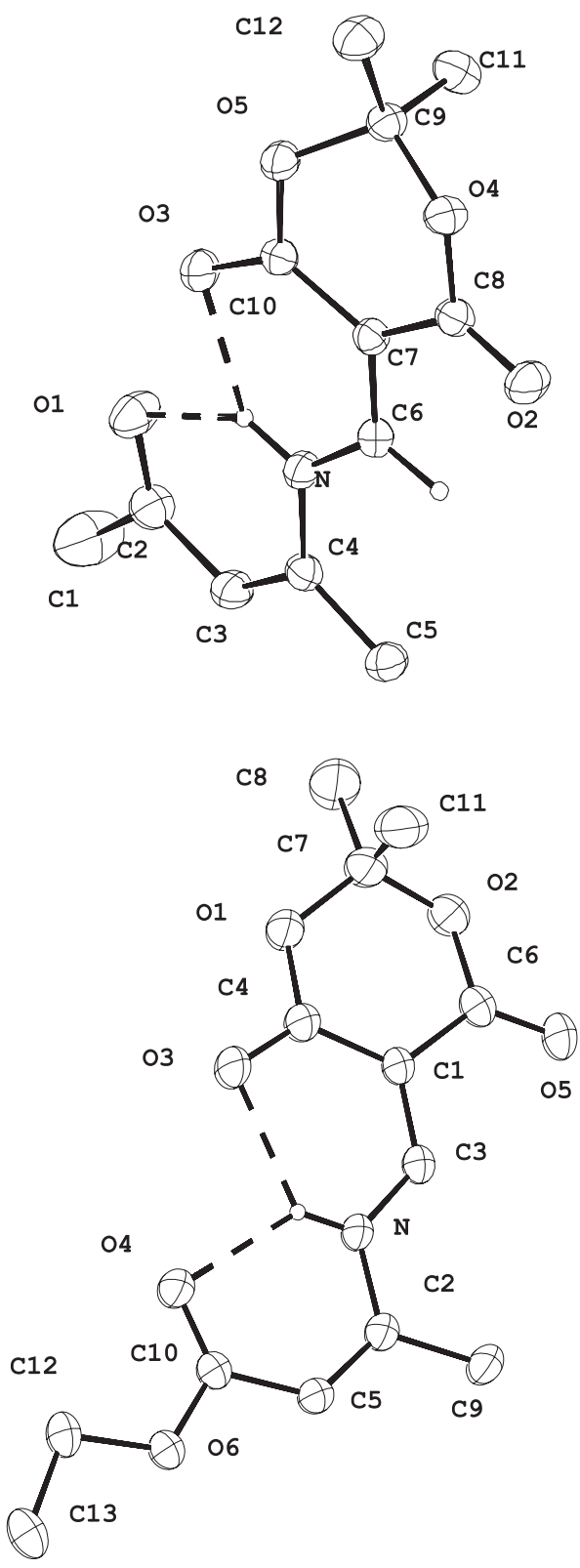

Figure 1. X-ray crystallographic structures of compounds 14a (top) and 14b (bottom). Displacement ellipsoids are shown at the $30 \%$ probability level. Only the $\mathrm{H}$-atoms involved in $\mathrm{H}$-bonds are shown with arbitrary size. The intramolecular H-bonds are shown with broken lines.

state is of particular interest to recognize the relationships between structural features and pharmacological properties, e.g. the anticonvulsant activity of enaminones has been associated with the inter- and intramolecular $\mathrm{NH} . . \mathrm{O}, \mathrm{CH} . . \mathrm{O}$ and $\mathrm{CH} . . . \mathrm{N}$ hydrogen bonding in the threedimensional structure. ${ }^{11}$ To unambiguously assign the structure of the obtained enaminones and to gain insight into intra- and intermolecular interactions the crystal structures of 14a and $14 \mathbf{b}$ were determined, and several structural features emerged. As noted in Figure 1, which

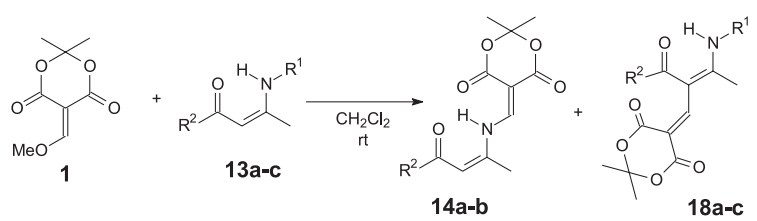

$\mathrm{R}^{1}=\mathrm{H}, \mathrm{R}^{2}=\mathrm{CH}_{3} \quad 14 \mathrm{a}, 40 \% ; 18 \mathrm{a}, 21 \%$ $\mathrm{R}^{1}=\mathrm{H}, \mathrm{R}^{2}=$ OEt $14 \mathrm{~b}, 33 \% ; 1 \mathbf{1 8}, 15 \%$ $\mathrm{R}^{1}=\mathrm{CH}_{2} \mathrm{CO}_{2} \mathrm{Et}, \mathrm{R}^{2}=\mathrm{CH}_{3} \quad 18 \mathrm{c}, 67 \%$

Scheme 2.

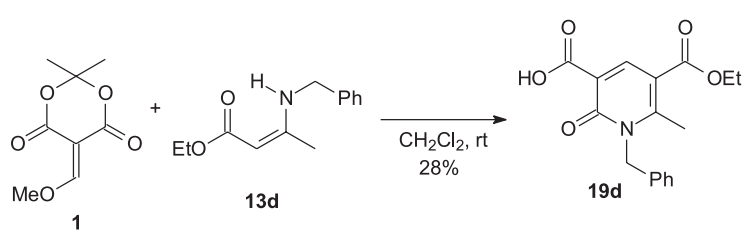<smiles>CCOC(=O)CN/C(C)=C(/C=C1C(=O)OC(C)(C)OC1=O)C(C)=O</smiles>

Scheme 3.

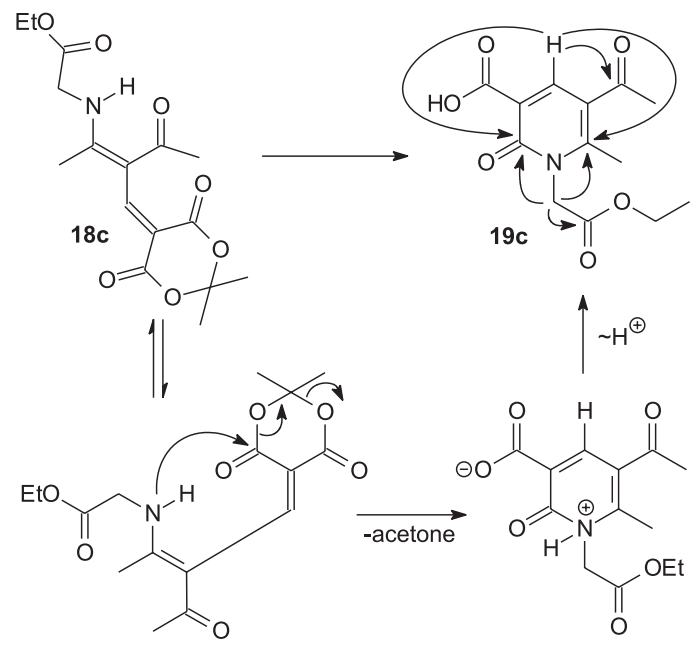

Scheme 4.

shows the molecules with labeled atoms, 14a has one strong bifurcated intramolecular hydrogen bonding between the oxygen atoms $\mathrm{O} 1$ and $\mathrm{O} 3$ and the $\mathrm{NH}$ group: $\mathrm{N}-\mathrm{H} 1 \mathrm{~N} . . . \mathrm{O} 1$ [2.699(3) $\AA$ ] and N-H1N...O3 [2.720(3) $\AA]$ ) providing two quasi-planar pseudo six-membered rings. The major distance from the least-square plane including all the atoms of these pseudo-rings is $0.187(3) \AA$ for atom O3. In addition, a weak C-H...O intermolecular H-bond was observed: C6H6...O3 $[1 / 2-\mathrm{x},-1 / 2+\mathrm{y}, \mathrm{z}, 3.379(3) \AA]$. The Meldrum's acid moiety has an envelope conformation, as can be seen from 
the Cremer and Pople ${ }^{12}$ parameters: $\mathrm{O} 4 \rightarrow \mathrm{C} 8 \rightarrow \ldots \mathrm{C} 9$ $\left[\mathrm{Q}=0.404(2) \AA, \theta=61.0(3)^{\circ}, \phi=299.0(4)^{\circ}\right]$. The $\mathrm{C} 9$ atom is 0.562 (3) $\AA$ out of the plane defined by the other atoms of the ring. In a similar way, $\mathbf{1 4 b}$ has a weak bifurcated intramolecular hydrogen bonding between the oxygen atoms $\mathrm{O} 3$ and $\mathrm{O} 4$ and the $\mathrm{NH}$ group: N-H2...O3 [2.763(2) $\AA]$ and N-H2...O4 [2.753(2) A] , also providing two quasiplanar pseudo six-membered rings. Here again, a weak $\mathrm{C}$ H...O intermolecular H-bond was also noted: C13H13B...O3' [1-x, y, 1-z, 3.416(3) ̊].

In conclusion, the reactions of acyclic enaminones and methoxymethylene Meldrum's acid afford $N$ - and/or $C$ adducts and 2-pyridones were formed from the latter through an intramolecular aza-annulation. The scope, limitations and the application of the methodology here described in natural products synthesis is under investigation in our lab and will be reported opportunely.

\section{Experimental}

Melting points were determined on a Karl Kolb apparatus and are uncorrected. Infrared spectra were recorded as $\mathrm{KBr}$ discs on a FT-IR BOMEM MB100 instrument. NMR spectra were obtained for ${ }^{1} \mathrm{H}$ at $300 \mathrm{MHz}$ and for ${ }^{13} \mathrm{C}$ at $75 \mathrm{MHz}$ using a Varian Gemini 300 or a Bruker AC300P spectrometers at Instituto de Química, UNICAMP. Chemical shifts are reported in ppm units downfield from reference (internal TMS). MS spectra were measured on a SHIMADSU CG-MS QP-5050 spectrometer at $70 \mathrm{eV}$. Elemental analyses were performed on a 2400 CHN Perkin Elmer instrument at Instituto de Química, UNICAMP. Enaminones 13a-b, ${ }^{6}$ 13c, ${ }^{13}$ 13d, ${ }^{6}$ Meldrum's $\operatorname{acid}^{13}$ and methoxymethylene Meldrum's acid ${ }^{13}$ were prepared according to known procedures. The single crystal $\mathrm{X}$-ray data collections were carried out on a Nonius CAD4 diffractometer at Departamento de Química, UFSC.

\section{General synthetic procedure}

A solution of $2 \mathrm{mmol}$ of Meldrum's acid in $2 \mathrm{~mL}$ of trimethyl orthoformate was heated at reflux for $2 \mathrm{~h}$ after which time the solvent was evaporated. The solid that formed was dissolved in $5 \mathrm{~mL}$ of $\mathrm{CH}_{2} \mathrm{Cl}_{2}$ and $2 \mathrm{mmol}$ of enaminone was added and the solution was allowed to stand at room temperature for $24 \mathrm{~h}$. The solvent was evaporated and the crude residue was treated as indicated in each case.

2, 2 -dime thyl-5-[(Z)-1-methyl-3-oxo-1butenylaminomethylene]-1,3-dioxane-4,6-dione (14a).Purified by silica gel column chromatography (benzene/ethyl acetate 20\%), pale orange needles, mp 193- $196^{\circ} \mathrm{C}$. IR (KBr): $v_{\max } / \mathrm{cm}^{-1} 3079,1736,1690,1664,1600$, 1580. ${ }^{1} \mathrm{H} \mathrm{NMR}\left(\mathrm{CDCl}_{3}\right): 1.74(6 \mathrm{H}, \mathrm{s}) ; 2.22(3 \mathrm{H}, \mathrm{s}) ; 2.26(3 \mathrm{H}$, s); $5.75(1 \mathrm{H}, \mathrm{s}) ; 8.35$ (1H, d, J 14.3Hz); 13.93 (1H, br s). ${ }^{13} \mathrm{C}$ NMR $\left(\mathrm{CDCl}_{3}\right)$ : $18.1\left(\mathrm{CH}_{3}\right) ; 27.3\left(\mathrm{CH}_{3}\right) ; 30.8\left(\mathrm{CH}_{3}\right) ; 90.8$ (C); $105.0(\mathrm{C}) ; 109.7(\mathrm{CH}) ; 148.4(\mathrm{C}) ; 150.8(\mathrm{CH}) ; 162.8$ (C); 163.7 (C); 199.0 (C). MS, m/z (\%): 253 [M+, 41\%], 195 (100\%), 149 (99\%); $136(56 \%), 108$ (37\%). Anal. Calcd. for $\mathrm{C}_{12} \mathrm{H}_{15} \mathrm{NO}_{5}: \mathrm{C}, 56.90 \%$; H, 5.95\%; N, 5.55\%. Found: C, $56.97 \%$; H, 6.01\%; N, 5.54\%.

5-[(Z)-2-acetyl-3-amino-2-butenylidene ]-2,2-dimethyl1,3-dioxane-4,6-dione (18a). Purified by silica gel column chromatography (benzene/ethyl acetate $50 \%$ ), pale yellow needles, mp 158-161 ${ }^{\circ} \mathrm{C}$. IR (KBr): $v_{\max } / \mathrm{cm}^{-1} 3420,3200$, 1728, 1690, 1631. ${ }^{1} \mathrm{H}$ NMR $\left(\mathrm{CDCl}_{3}\right): 1.75(6 \mathrm{H}, \mathrm{s}) ; 2.24$ $(3 \mathrm{H}, \mathrm{s}) ; 2.39(3 \mathrm{H}, \mathrm{s}) ; 7.97(1 \mathrm{H}$, br s$) ; 8.64(1 \mathrm{H}, \mathrm{s}) ; 10.60$ (1H, br s). ${ }^{13} \mathrm{C} \mathrm{NMR}\left(\mathrm{CDCl}_{3}\right): 21.5\left(\mathrm{CH}_{3}\right) ; 27.4\left(\mathrm{CH}_{3}\right) ; 28.5$ $\left(\mathrm{CH}_{3}\right) ; 99.7(\mathrm{C}) ; 103.5(\mathrm{C}) ; 110.9(\mathrm{C}) ; 155.1(\mathrm{CH}) ; 163.0$ (C); 164.6 (C); 172.4 (C); 199.4 (C). MS, m/z (\%): 253 [M+, 70\%], 195 (48\%), 151 (97\%), 149 (37\%), $136(57 \%)$, 123 (84\%), 108 (100\%), 95 (65\%), 80 (75\%). Anal. Calcd. for $\mathrm{C}_{12} \mathrm{H}_{15} \mathrm{NO}_{5}: \mathrm{C}, 56.90 \% ; \mathrm{H}, 5.95 \%$; N, 5.55\%. Found: C, $56.97 \%$; H, 6.01\%; N, 5.54\%.

Ethyl (Z)-3-(2,2-dimethyl-4,6-dioxo-1,3-dioxan-5ylidenmethylamino)-2-butenoate (14b). Purified by silica gel column chromatography (benzene/ethyl acetate 20\%), colorless solid, mp 193-194 ${ }^{\circ} \mathrm{C}$. IR (KBr): $v_{\max } / \mathrm{cm}^{-1} 3136$, $1741,1698,1651,1603 \mathrm{~cm}^{-1} .{ }^{1} \mathrm{H}$ NMR $\left(\mathrm{CDCl}_{3}\right): 1.31(3 \mathrm{H}$, $\mathrm{t}, J 7.1 \mathrm{~Hz}) ; 1.74(6 \mathrm{H}, \mathrm{s}) ; 2.22(3 \mathrm{H}, \mathrm{s}) ; 4.31(2 \mathrm{H}, \mathrm{q}, J 7.1 \mathrm{~Hz})$; $5.37(1 \mathrm{H}, \mathrm{s}) ; 8.32(1 \mathrm{H}, \mathrm{d}, J 14.3 \mathrm{~Hz}) ; 13.39\left(1 \mathrm{H}\right.$, br s). ${ }^{13} \mathrm{C}$ NMR $\left(\mathrm{CDCl}_{3}\right)$ : $14.2\left(\mathrm{CH}_{3}\right) ; 18.1\left(\mathrm{CH}_{3}\right) ; 27.1\left(\mathrm{CH}_{3}\right) ; 60.7$ $\left(\mathrm{CH}_{2}\right) ; 90.1(\mathrm{C}) ; 103.0(\mathrm{CH}) ; 105.1(\mathrm{C}) ; 148.8(\mathrm{C}) ; 150.5$ $(\mathrm{CH}) ; 163.5$ (C); 163.9 (C); 167.1 (C). MS, m/z (\%): 283 $\left[\mathrm{M}^{+}, 22 \%\right], 259$ (22\%), 225 (71\%), 196 (100\%). Anal. Calcd. for $\mathrm{C}_{13} \mathrm{H}_{17} \mathrm{NO}_{6}$ : C, 55.12\%; H, 6.01\%; N, $4.95 \%$. Found: C, 54.83\%; H, 5.96\%; N, 4.32\%.

Ethyl (Z)-3-amino-2-(2,2-dimethyl-4,6-dioxo-1,3dioxan-5-ylidenmethyl)-2-butenoate (18b). Purified by silica gel column chromatography (benzene/ethyl acetate $30 \%$ ), yellow needles, mp $172-174^{\circ} \mathrm{C}$. IR (KBr): $v_{\max } / \mathrm{cm}^{-1}$ 3317, 3109, 1725, 1668. ${ }^{1} \mathrm{H}$ NMR $\left(\mathrm{CDCl}_{3}\right): 1.23(3 \mathrm{H}, \mathrm{t}, J$ $7.1 \mathrm{~Hz}) ; 1.66(6 \mathrm{H}, \mathrm{s}) ; 2.38(3 \mathrm{H}, \mathrm{s}) ; 4.17(2 \mathrm{H}, \mathrm{q}, J 7.1 \mathrm{~Hz})$; $7.04\left(1 \mathrm{H}\right.$, br s); $8.40(1 \mathrm{H}, \mathrm{s}) ; 9.38\left(1 \mathrm{H}\right.$, br s). ${ }^{13} \mathrm{C} \mathrm{NMR}$ $\left(\mathrm{CDCl}_{3}\right): 14.4\left(\mathrm{CH}_{3}\right) ; 21.0\left(\mathrm{CH}_{3}\right) ; 27.4\left(\mathrm{CH}_{3}\right) ; 60.7\left(\mathrm{CH}_{2}\right)$; $101.6(\mathrm{C}) ; 101.7(\mathrm{C}) ; 103.8(\mathrm{C}) ; 153.5(\mathrm{CH}) ; 163.3(\mathrm{C})$; 165.5 (C); 169.2 (C); 170.8 (C). MS, m/z (\%): $283\left[\mathrm{M}^{+}\right.$, 48\%], 225 (36\%), 181 (100\%), 153 (91\%), 136 (31\%), 124 (57\%). Anal. Calcd. for $\mathrm{C}_{13} \mathrm{H}_{17} \mathrm{NO}_{6}: \mathrm{C}, 55.12 \%$; H, 6.01\%; N, $4.95 \%$. Found: C, 55.23\%; H, 6.07\%; N, $4.72 \%$.

Ethyl 2-[(Z)-2-(2,2-dimethyl-4,6-dioxo-1,3-dioxan-5ylidenmethyl)-1-methyl-3-oxo-1-butenylamino]acetate (18c). Recrystallized from $\mathrm{CH}_{2} \mathrm{Cl}_{2}$ /petroleum ether, yellow 
solid, mp 139-142 ${ }^{\circ} \mathrm{C} . \mathrm{IR}(\mathrm{KBr}): v_{\max } / \mathrm{cm}^{-1} 3224,1736,1690$, 1647. ${ }^{1} \mathrm{H} \mathrm{NMR}\left(\mathrm{CDCl}_{3}\right): 1.34(3 \mathrm{H}, \mathrm{t}, J 7.1 \mathrm{~Hz}) ; 1.74(6 \mathrm{H}, \mathrm{s})$; $2.22(3 \mathrm{H}, \mathrm{s}) ; 2.42(3 \mathrm{H}, \mathrm{s}) ; 4.26(2 \mathrm{H}, \mathrm{d}, J 5.1 \mathrm{~Hz}) ; 4.32(2 \mathrm{H}, \mathrm{q}$, $J 7.1 \mathrm{~Hz}) ; 8.72(1 \mathrm{H}, \mathrm{s}) ; 12.64(1 \mathrm{H}$, br s $) .{ }^{13} \mathrm{C} \mathrm{NMR}\left(\mathrm{CDCl}_{3}\right)$ : $14.1\left(\mathrm{CH}_{3}\right) ; 18.6\left(\mathrm{CH}_{3}\right) ; 27.4\left(\mathrm{CH}_{3}\right) ; 27.7\left(\mathrm{CH}_{3}\right) ; 46.1\left(\mathrm{CH}_{2}\right)$; $62.7\left(\mathrm{CH}_{2}\right) ; 99.3(\mathrm{C}) ; 103.3(\mathrm{C}) ; 111.2(\mathrm{C}) ; 154.9(\mathrm{CH})$; 162.3 (C); 164.5 (C); $166.6(\mathrm{C}) ; 173.5$ (C); 197.5 (C). MS, $\mathrm{m} / \mathrm{z}(\%): 339\left[\mathrm{M}^{+}, 34 \%\right], 281$ (62\%), 237 (56\%), 135 (100\%). Anal. Calcd. for $\mathrm{C}_{16} \mathrm{H}_{21} \mathrm{NO}_{7} \mathrm{C}, 56.64 \%$; $\mathrm{H}, 6.19 \%$; N, 4.13\%. Found: C, 56.47\%; H, 6.09\%; N, $4.04 \%$.

5-acetyl-1-ethyloxycarbonylmethyl-6-methyl-2-oxo1,2-dihydro-3-pyridinecarboxylic acid $(\mathbf{1 9 c})$. A solution of $85.1 \mathrm{mg}(0.25 \mathrm{mmol})$ of $\mathbf{1 8 c}$ in $10 \mathrm{~mL}$ of toluene was heated at reflux for $24 \mathrm{~h}$, after which time the solvent was evaporated and the residue was recrystallized from $\mathrm{CH}_{2} \mathrm{Cl}_{2} /$ petroleum ether to give $52.9 \mathrm{mg}$ (75\% yield) of $19 \mathrm{c}$ as colorless needles, mp 121-123 ${ }^{\circ} \mathrm{C} .{ }^{1} \mathrm{H}$ NMR $\left(\mathrm{CDCl}_{3}\right): 1.34$ $(3 \mathrm{H}, \mathrm{t}, J 7.1 \mathrm{~Hz}), 2.62(3 \mathrm{H}, \mathrm{s}), 2.74(3 \mathrm{H}, \mathrm{s}), 4.31(2 \mathrm{H}, \mathrm{q}, J$ $7.1 \mathrm{~Hz}), 5.03(2 \mathrm{H}, \mathrm{s}), 8.90(1 \mathrm{H}, \mathrm{s}), 13.42(1 \mathrm{H}, 1) .{ }^{13} \mathrm{C} \mathrm{NMR}$ $\left(\mathrm{CDCl}_{3}\right): 14.1\left(\mathrm{CH}_{3}\right), 18.4\left(\mathrm{CH}_{3}\right), 29.6\left(\mathrm{CH}_{3}\right), 46.4\left(\mathrm{CH}_{2}\right)$, $62.8\left(\mathrm{CH}_{2}\right), 113.9(\mathrm{C}), 119.7(\mathrm{C}), 145.3(\mathrm{CH}), 157.3(\mathrm{C})$, 163.9 (C), 164.3 (C), 166.1 (C), 196.4 (C). MS, $m / z(\%)$ : $281\left[\mathrm{M}^{+}, 43 \%\right], 207$ (100\%). Anal. Calcd. for $\mathrm{C}_{13} \mathrm{H}_{15} \mathrm{NO}_{6} \mathrm{C}$, $55.52 \%$; H, 5.34\%; N, 4.98\%. Found: C, 55.44\%; H, 5.19\%; $\mathrm{N}, 5.14 \%$.

1-benzyl-5-ethyloxycarbonyl-6-methyl-2-oxo-1,2dihydro-3-pyridinecarboxylic acid (19d). Recrystallized from $\mathrm{CH}_{2} \mathrm{Cl}_{2}$ /petroleum ether, colorless solid, mp 135$137{ }^{\circ} \mathrm{C}$. IR (KBr): $v_{\max } / \mathrm{cm}^{-1} 1735,1720,1670,1620 .{ }^{1} \mathrm{H}$ $\mathrm{NMR}\left(\mathrm{CDCl}_{3}\right): 1.38(3 \mathrm{H}, \mathrm{t}, J 7.2 \mathrm{~Hz}), 2.86(3 \mathrm{H}, \mathrm{s}), 4.35(2 \mathrm{H}$, q, J 7.2Hz), $5.55(2 \mathrm{H}, \mathrm{s}), 7.11-7.15(2 \mathrm{H}, \mathrm{m}), 7.32-7.42(3 \mathrm{H}$, m), 9.07 (1H, s), 13.95 (1H, br s).

Crystal structure of 14a. $\mathrm{C}_{12} \mathrm{H}_{15} \mathrm{NO}_{5}, \mathrm{M}_{\mathrm{w}}=253.25$, orthorhombic, space group Pbca [nr. 61], $\mathrm{Z}=8$, a = 14.321(3), $\mathrm{b}=9.261(2), \mathrm{c}=19.064(4) \AA, \mathrm{V}=2528.4(9) \AA{ }^{3}{ }^{3}$ $\mathrm{d}_{\mathrm{c}}=1.331 \mathrm{Mg} \mathrm{m}^{-3}, \lambda($ Mo K $\alpha)=0.71073 \AA, \mu=0.104 \mathrm{~mm}^{-1}$, 2835 measured reflections, 2473 unique $\left(\mathrm{R}_{\mathrm{int}}=0.0\right)$ of which 1579 were considered as observed with $I \geq 2 \sigma(\mathrm{I})$. The single crystals were obtained by diffusion of petroleum ether into a solution of $\mathbf{1 4 a}$ in $\mathrm{CH}_{2} \mathrm{Cl}_{2}$ at room temperature.

Crystal structure of $\mathbf{1 4 b} . \mathrm{C}_{13} \mathrm{H}_{17} \mathrm{NO}_{6}, \mathrm{M}_{\mathrm{w}}=283.28$, monoclinic, space group $\mathrm{C} 2 / \mathrm{m}$ [nr. 12], $\mathrm{Z}=4, \mathrm{a}=18.015(4)$, $\mathrm{b}=6.600(1), \mathrm{c}=12.056(2) \AA, \mathrm{V}=1430.7(5) \AA,{ }^{3} \mathrm{~d}_{\mathrm{c}}=1.315$ $\mathrm{Mg} \mathrm{m}^{-3}, \lambda($ Mo K $\alpha)=0.71073 \AA$ A $\mu=0.105 \mathrm{~mm}^{-1}, 3066$ measured reflections, 1535 unique $\left(\mathrm{R}_{\text {int }}=0.016\right)$ of which 1225 were considered as observed with $\mathrm{I} \geq 2 \sigma(\mathrm{I})$. The molecule is placed on the mirror symmetry plane, except the atoms $\mathrm{O} 1, \mathrm{O} 2, \mathrm{C} 8$ and $\mathrm{C} 11$, that are disordered. The single crystals were obtained by diffusion of petroleum ether into a solution of $\mathbf{1 4 b}$ in $\mathrm{CH}_{2} \mathrm{Cl}_{2}$ at room temperature. The structures were solved with direct methods using
SHELXS97 ${ }^{14}$ and refined anisotropically with full-matrix least-squares on $\mathrm{F}^{2}$ using SHELXL97. ${ }^{15}$ The hydrogen atoms were placed at calculated position except those involved in H-bonds, found on difference maps and refined. Final indices: $\mathrm{R}_{1}\left(\mathrm{~F}_{\mathrm{o}}\right)=0.043, \mathrm{wR}_{2}\left(\mathrm{~F}^{2}\right)=0.131$ for 172 refined parameters and $\mathrm{R}_{1}\left(\mathrm{~F}_{\mathrm{o}}\right)=0.048, \mathrm{w}_{2}\left(\mathrm{~F}^{2}\right)=0.128$ for 145 refined parameters, for $\mathbf{1 4 a}$ and $\mathbf{1 4 b}$, respectively.

The crystallographic data (excluding structure factors) for structures in this paper have been deposited with the Cambridge Crystallographic Data Centre as supplementary publication numbers CCDC 179109 and CCDC 179108, for 14a and 14b, respectively. Copies of the data can be obtained, free of charge via www.ccdc.cam.ac.uk/conts/retrieving.html (or from the Cambridge Crystallographic Data Centre, CCDC, 12 Union Road, Cambridge, CB21EZ, UK (fax +44 1223 336033) or e-mail: deposit@ccdc.camac.uk.)

\section{Acknowledgments}

The authors thank the Brazilian Agencies for fellowships to V.C.S. (PIBIC/CNPq), H.B.N. (CAPES), I.V. (CNPq) and financial support from FUNAPE-UFG. The authors also thank Instituto de Química, UNICAMP (NMR and elemental analyses), Departamento de Química, UFSCar (NMR) for measurements and Departamento de Química, UFSC for the X-ray single crystal data collections.

\section{References}

1. Lue, P.; Greenhill, J. V. In Advances in Heterocyclic Chemistry; Katritzky, A. R., ed.; Academic Press: New York, 1997, vol. 67, pp 207-343; Kuckländer, V. In The Chemistry of Enamines; Rappoport, Z., ed.; John Wiley \& Sons: New York, 1994; Part 1, pp 525-639.

2. Cheng, Y.; Yang, H.-B.; Huang, Z.-T.; Wang, M.-X.; Tetrahedron 2001, 42, 1757; Adlington, R. M.; Baldwin, J. E.; Catterick, D.; Pritchard, G. J.; Tang, L. T.; J. Chem. Soc., Perkin Trans. 1 2000, 2311; Hsung, R. P.; Wei, L.-L.; Sklenicka, H. M.; Douglas, C. J.; McLaughlin, M. J.; Mulder, J. A.; Yao, L. J.; Org. Lett. 1999, 1, 509; Alberola, A.; Calvo, L. A.; Ortega, A. G.; Ruíz, M. C. S.; Yustos, P.; J. Org. Chem. 1999, 64, 9493; Risi, C. D.; Pollini, G. P.; Veronese, A. C.; Bertolasi, V.; Tetrahedron Lett. 1999, 40, 6995; Ferraz, H. M. C.; Pereira, F. L. C.; Leite, F. S.; Nunes, M. R. S.; Payret-Arrua, M. E.; Tetrahedron 1999, 55, 10915; d'Angelo, J.; Cavé, C.; Desmaële, D.; Gassama, A.; Thominiaux, C.; Riche, C.; Heterocycles 1998, 47, 725; Kascheres, A.; Schumacher, H. C.; Rodrigues, R. A. F.; J. Heterocyclic Chem. 1997, 34, 757; Kascheres, A.; Rodrigues, R. A. F.; Tetrahedron 1996, 52, 12919; Ferraz, H. M. C.; Oliveira, E. O.; Payret-Arrua, M. E.; Brandt, C. A.; J. Org. Chem. 1995, 60, 7357. 
3. Benovsky, P.; Stepheson, G. A.; Stille, J. R.; J. Am. Chem. Soc 1998, 120, 2493; Barta, N. S.; Brode, A.; Stille, J. R.; J. Am. Chem. Soc. 1994, 116, 6201; Michael, J. P.; Gravestock, D.; J. Chem. Soc., Perkin Trans. 1 2000, 1919; Michael, J. P.; Koning, C. B.; Gravestock, D.; Hosken, G. D.; Howard, A. S.; Jungmann, C. M.; Krause, R. W. M.; Parsons, A. S.; Pelly, S. C.; Stanbury, T. V.; Pure Appl. Chem. 1999, 71, 979; Michael, J. P.; Gravestock, D.; Eur. J. Org. Chem. 1998, 865; Michael, J. P.; Gravestock, D.; Pure Appl. Chem. 1997, 69, 583; Desmaële, D.; Mekouar, K.; d'Angelo, J.; J. Org. Chem. 1997, 62, 3890; Paulvannan, K.; Stille, J. R.; J. Org. Chem. 1994, 59, 1613; Cook, G. R.; Beholz, L. G.; Stille, J. R.; Tetrahedron Lett. 1994, 35, 1669; Paulvannan, K.; Stille, J. R.; Tetrahedron Lett. 1993, 34, 6673; Paulvannan, K.; Schwarz, J. B.; Stille, J. R.; Tetrahedron Lett. 1993, 34, 215.

4. Benovsky, P.; Stepheson, G. A.; Stille, J, R.; J. Am. Chem. Soc. 1999, 120, 2493; Beholz, L. G.; Benovsky, P.; Ward, D. L.; Barta, N. S.; Stille, J, R; J. Org. Chem. 1997, 62, 1033; Smith III, A. B.; Guzman, M. C.; Sprengeler, P. A.; Keenan, T. P.; Holcomb, R. C.; Wood, J. L.; Carrol, P. J.; Hirschann, R.; J. Am. Chem. Soc. 1994, 116, 9947; Paulvannan, K.; Stille, J. R.; Tetrahedron Lett. 1993, 34, 8197.

5. Katritzky, A. R.; Fang, Y.; Donkor, A.; Xu, J.; Synthesis 2000, 2029; Wisniewski Junior, A.; Oliveira, A. R. M.; Cunha, C. J.; Simonelli, F.; Marques, F. A.; J. Braz. Chem. Soc. 1999, 10, 369; Fustero, S.; Torre, M. G.; Pina, B.; Fuentes, A. S.; J. Org. Chem. 1999, 64, 5551; Fustero, S.; Torre, M. G.; Jofré, V.; Carlon, P. Navarro, A.; Fuentes, A. S.; Carrió, J. S.; J. Org Chem. 1998, 63, 8825; Seko, S.; Tani, N.; Tetrahedron Lett. 1998, 39, 8117; Bartoli, G. Cimarelli, C.; Palmieri, G. Bosco, M.; Dalpozzo, R.; Synthesis 1990, 895.

6. Valduga, C. J.; Squizani, A.; Braibante, H. S.; Braibante, M. E. F.; Synthesis 1998, 1019; Braibante, M. E. F.; Braibante, H. S.; Missio, L.; Andricopulo, A.; Synthesis 1994, 898; Braibante, M. E. F.; Braibante, H. S.; Salvatore, S. J. S. A.; Quim. Nova 1990, 13, 67 .
7. Valduga, C. J.; Santis, D. B.; Braibante, H. S.; Braibante, M. E. F.; J. Heterocyclic Chem. 1998, 36, 505; Valduga, C. J.; Braibante, H. S.; Braibante, M. E. F.; J. Heterocyclic Chem. 1997, 34, 1453; Missio, L. J.; Braibante, H. S.; Braibante, M. E. F.; J. Heterocyclic Chem. 1996, 33, 1243.

8. McNab, H.; Monahan, L. C.; J. Chem. Soc., Perkin Trans. 1 1989, 419; Gatta, F.; Giudice, M. R. D.; Pomponi, M.; Marta, M.; Heterocycles 1992, 34, 991; Derbyshire, P. A.; Hunter, G. A.; McNab, H.; Monahan, L. C.; J. Chem. Soc., Perkin Trans. 1 1993, 2017.

9. Zhuo, J.-C.; Magn. Reson. Chem. 1998, 36, 565.

10. Decicco, C.; Grover, P.; J. Org. Chem. 1996, 61, 3534; Chen, B.-C.; Heterocycles 1991, 32, 529.

11. Kubicki, M.; Bassyouni, H. A. R.; Codding, P. W.; J. Mol. Struct. 2000, 525, 141; Foster, J. E.; Nicholson, J. M.; Butcher, R.; Stables, J. P.; Edafiogho, I. O.; Goodwin, A. M.; Henson, M. C.; Smith, C. A.; Scott, K. R.; Bioorg. Med. Chem. 1999, 7, 2415.

12. Cremer, D.; Pople, J. A.; J. Am. Chem. Soc. 1975, 97, 1354.

13. Rechsteiner, B.; Texier-Boullet, F.; Hamelin, J.; Tetrahedron Lett. 1993, 34, 5071; Davison, D.; Berhard, S. A.; J. Am. Chem. Soc. 1948, 70, 3426; Jourdain, F.; Pommelet, J. C.; Synth. Commun. 1997, 27, 483.

14. Sheldrick, G. M.; SHELXS97; Program for the Solution of Crystal Structures; University of Göttingen, Germany, 1990.

15. Sheldrick, G. M.; SHELXL97; Program for the Refinement of Crystal Structures; University of Göttingen, Germany, 1997.

Received: May 5, 2002

Published on the web: February 12, 2003 